\title{
Kabylie : Economie ancienne ou traditionnelle
}

\section{S. Doumane}

\section{OpenEdition}

Journals

Édition électronique

URL : http://journals.openedition.org/encyclopedieberbere/1419

DOI : 10.4000/encyclopedieberbere.1419

ISSN : 2262-7197

\section{Éditeur}

Peeters Publishers

\section{Édition imprimée}

Date de publication : 1 mai 2004

Pagination : 4034-4038

ISBN : 2-7449-0452-X

ISSN : 1015-7344

\section{Référence électronique}

S. Doumane, « Kabylie : Economie ancienne ou traditionnelle », Encyclopédie berbère [En ligne], 26। 2004, document K10, mis en ligne le 01 juin 2011, consulté le 14 décembre 2020. URL : http:// journals.openedition.org/encyclopedieberbere/1419; DOI : https://doi.org/10.4000/ encyclopedieberbere.1419

Ce document a été généré automatiquement le 14 décembre 2020.

(c) Tous droits réservés 


\section{Kabylie : Economie ancienne ou traditionnelle}

\section{S. Doumane}

1 On entend par économie kabyle ancienne ou traditionnelle le mode de production, d'échange et de consommation encore en vigueur en Kabylie à la fin du $\mathrm{xIX}^{\mathrm{e}}$ siècle $^{1}$. En rendre compte de façon exhaustive n'est pas aisé en raison de deux contraintes :

- La première, d'ordre théorique et méthodologique, est liée à la faiblesse sinon l'absence d'études approfondies sur l'histoire économique de cette région montagneuse d'Algérie.

- La deuxième, plus empirique, est inhérente à la fragmentation des informations et des données chiffrées collectées et rapportées par divers auteurs (militaires, administrateurs, anthropologues...) dans la seconde moitié $d u$ XIX ${ }^{e}$ siècle pour l'essentiel.

2 À défaut donc de décrire précisément les rouages de l'économie kabyle traditionnelle, on se contentera de donner un aperçu de ses principales caractéristiques.

\section{Une économie de subsistance d'apparence aberrante}

3 Jusqu'à la veille de la guerre d'indépendance (1954-1962), persistaient encore en Kabylie des restes d'activités anciennes témoignant d'une certaine vitalité économique de la région. On pourrait qualifier cette économie de "kabyle ", dans la mesure où elle était fondée sur des facteurs humains et matériels endogènes. Mais la disponibilité de ces facteurs était toute relative et se caractérisait par un déséquilibre flagrant; d'un côté une densité humaine importante, de l'autre des ressources naturelles dont la principale d'entre elles, la terre, rares ; à tel point «qu'on a qualifié d'absurde l'économie kabyle traditionnelle» (Mammeri 1980, p. 32).

4 Ce déséquilibre entre la forte densité démographique et la pauvreté en ressources naturelles du pays kabyle ne date pas du xix ${ }^{e}$ siècle ; Ibn Khaldoun signalait déjà en son 
temps la présence d'une population nombreuse dans le massif situé entre Dellys et Bougie.

5 Toujours est-il qu'à la veille de la colonisation française prévalait en Kabylie une économie de type essentiellement vivrier, mise en œuvre dans un espace restreint et montagneux à $90 \%$, mobilisant une main d'œuvre abondante et exploitant des ressources naturelles très limitées.

6 Nécessairement fragile, car sensible aux aléas naturels (sécheresse, invasion de sauterelles...) et socio-politiques (conflits, guerres...), un tel système de production ne pouvait être viable et douée d'une certaine efficacité qu'incrusté dans une organisation sociale adéquate. Précisément, le mode de production kabyle traditionnel était inconcevable séparé de son substrat sociologique: une organisation lignagère fortement liée à la terre et dont la survie dépendait de sa capacité à mobiliser toutes ses ressources, matérielles et humaines, sous la houlette d'un chef de famille obnubilé par la cohésion et la reproduction de son groupe (cf. Bourdieu 1972).

7 La pratique agricole tenait une place importante dans ce système socio-économique mais elle ne pouvait à elle seule suffire à subvenir aux besoins de la communauté. Elle était au centre d'une kyrielle d'autres activités économiques : petites industries (forge, armurerie, bijouterie, moulins...), artisanat (poterie, vannerie, tissage...), petit commerce (souk, colportage...).

8 L'équilibre global de cette économie reposait sur une sorte de division du travail et un flux d'échanges entre la montagne et la plaine, le piémont et les villes environnantes. En période de paix, ces rapports étaient relativement fructueux et profitaient à l'économie kabyle 2 .

9 Si on ne considère pas ce système complexe et séculaire d'échanges entre la Kabylie montagnarde et son environnement immédiat et parfois lointain, on ne peut saisir la densité (paradoxale ?) de son peuplement, de son organisation sociale et surtout de ses pratiques économiques ${ }^{3}$.

10 C'est la rupture de ce système par l'occupation militaire française à partir des années 1850, la répression et la confiscation des terres des vallées et du piémont lors des révoltes de 1857 et 1871, qui fut à l'origine du démantèlement de l'économie kabyle ancienne. Dès la fin du xIX ${ }^{e}$ siècle, l'agriculture coloniale et l'industrie française commencèrent à recruter des travailleurs par milliers, privant ainsi l'économie kabyle de sa principale substance.

\section{Les principales activités économiques traditionnelles}

\section{La terre et les activités agricoles}

11 À l'instar d'autres régions d'Algérie, la Kabylie pré-coloniale était une région rurale ${ }^{4}$ où la terre, aussi rare fût-elle, ne constituait pas moins le facteur de production essentiel autour duquel s'organisait la vie économique mais aussi sociale et culturelle. Le procès de travail s'exerçait dans le cadre du groupe familial et on ne recourait guère à la force de travail extérieure sauf dans le cas de l'entraide entre groupes familiaux (tiwizi) ${ }^{5}$. Peu pourvus en terres agricoles, les paysans kabyles s'évertuaient à mettre en valeur la moindre parcelle de terre cultivable; ils pratiquaient des combinaisons culturales où l'arbre et l'herbe jouaient un rôle important, ce qui leur permettait de produire des 
fruits (figues, glands, raisins...), de l'huile (huile d'olives) et d'élever des animaux (caprins, ovins, bovins) ${ }^{6}$. Autour des maisons et en contrebas des villages, toutes les familles entretenaient des jardins potagers (timizar) pour s'approvisionner en légumes (fèves, pommes de terre, oignons, lentilles...). Pour accéder à plus d'espace agricole, ils procédaient à des associations avec les propriétaires fonciers des plaines environnantes. Ces formes de coopération permettaient aux montagnards de se procurer les céréales (blé et orge) qui constituaient la base de leur consommation alimentaire.

\section{La manufacture et l'artisanat}

12 Si les Kabyles étaient d'abord des paysans voués au travail de la terre, ils s'adonnaient aussi à d'autres occupations nécessaires à la production de leurs moyens d'existence. Polyvalents, "la plupart des tribus et des villages fabriquaient eux-mêmes les objets indispensables aux activités quotidiennes [...], pourvoyaient aux besoins de la Kabylie tout entière et alimentaient également leur commerce avec l'extérieur " (Mahé 2001).

13 - La transformation du fer et des métaux.

$\mathrm{Au}$ début de la conquête de la Kabylie (1840-1850), les rapports militaires signalaient l'existence d'une industrie du fer et des métaux (Carette 1848) dans plusieurs tribus kabyles et quelques unes étaient même spécialisées ${ }^{7}$. En réalité, le travail du fer était une pratique fort répandue dans de nombreux villages car sa raison d'être première était de répondre aux besoins de la vie quotidienne, dont ceux inhérents à l'activité agricole (instruments aratoires, faucilles, pioches, haches...). A. Hanoteau et A. Letourneux, dans leur œuvre encyclopédique sur la Kabylie parue en 1873 firent état de l'habileté des ouvriers du fer (iheddaden), du bois et de la panoplie de produits de forge fabriqués (Hanoteau \& Letourneux 2003). Le capitaine Carette avait dénombré plus de 250 ateliers ou forges dont la plus grande partie était concentrée en Kabylie maritime (Azeffoun) et forestière (Akfadou) (Carette 1848).

14 - Autres activités industrielles et artisanales.

A côté des industries du fer et des métaux qui requéraient un certain savoir-faire technique et la disponibilité du minerai, pas toujours à la portée de tous, les Kabyles étaient fort pourvus d'autres activités manufacturières et artisanales dont ils dégageaient quelques surplus alimentant leur commerce. Les plus courantes utilisaient des matières premières disponibles sur place ou faciles à faire venir d'autres régions d'Algérie ou d'Afrique du Nord ; parmi celles-ci on peut citer :

Le travail du bois et la menuiserie: La présence d'une importante forêt jusqu'au XIX siècle avait permis de nombreuses activités liées à l'exploitation du bois. Aux nombreux produits destinés aux usages agricoles (charrues, manches pour l'outillage agricole...) et domestiques (métiers à tisser, ustensiles de cuisine...), s'ajoutaient des produits de menuiserie et d'art (portes, toitures, coffrets, meubles sculptés...) et, à certaines époques la coupe de certaines essences pour les chantiers navals (Ottomans...) et l'exportation (Tunisie, Égypte).

Les moulins et pressoirs à huile: On dénombrait à la fin du $\mathrm{XIX}^{\mathrm{e}}$ siècle de nombreux moulins et pressoirs à huile. Le capitaine Carette avait recensé plus de 600 moulins à eau dans les seuls cercles de Tizi-Ouzou et de Fort-National servant à triturer les grains et les légumineuses et à retenir l'eau nécessaire à d'autres activités artisanales: teintureries, tanneries... Quant aux huileries, il y en avait dans tous les villages, A. 
Hanoteau et A. Letourneux en avaient signalé presque un millier dans les cercles de Tizi-Ouzou, Draâ-El-Mizan, Dellys et Fort-National attestant que « la fabrication de l'huile d'olives est une des industries les plus importantes du pays » (2003, p. 414).

Le tissage et le travail de la laine: Activité artisanale essentiellement féminine, elle était présente dans tous les foyers ; « il n'est [...] de maison qui n'ait son métier» remarquaient Hanoteau et Letourneux (2003, p. 428). On confectionnait des vêtements pour hommes et femmes, des tapis, des couvertures et des burnous. Certains de ces produits tels les burnous et les tapis étaient appréciés dans les villes et se vendaient sur tous les souks d'Algérie et même d'Afrique du Nord. La tribu des Ait Abbas, déjà mentionnée, possédait des ateliers de lainage et était réputée pour la qualité de ses burnous.

La poterie, la vannerie, la sellerie et autres activités artisanales: Ignorant la spécialisation et la division du travail, l'économie kabyle traditionnelle avait pour vocation de répondre à l'essentiel des besoins économiques de la population. Certaines productions artisanales comme la poterie, la vannerie, la sellerie étaient aussi importantes que l'activité métallurgique ou le tissage mais de nombreuses autres activités n'étaient pas négligeables : fabrication du savon, de tamis, tuilerie, plâtrerie, travail de l'alfa et du palmier nain (doum)...

\section{Le commerce et les échanges}

19 Plus que tout autre indice, ce sont les échanges qui renseignent sur la vitalité de l'économie kabyle traditionnelle. "Si jamais ils parviennent à amasser de grands capitaux, ils s'initieront promptement à nos procédés et seront de rudes concurrents pour nos négociants " remarquaient Hanoteau et Letourneux à propos de l'habileté des Kabyles dans le commerce. Il y avait, en effet, un réseau dense de marchés à l'intérieur du pays kabyle (leswaq) - le capitaine Carette en avait recensé 67 se tenant dans la semaine ${ }^{8}$ - mais aussi un réseau extérieur (dans les grandes agglomérations algériennes, voire tunisiennes et marocaines) alimenté par des marchands kabyles (négociants de passage, comptoirs fixes...). À cela il faut ajouter les colporteurs, marchands modestes mais très nombreux ${ }^{9}$ et entreprenants, qui parcouraient des centaines de kilomètres à travers l'Algérie pour écouler les produits de leur artisanat et rapporter des produits qui manquaient en Kabylie.

"Les Kabyles importent dans leur pays du blé, de l'orge, des bœufs pour le labourage et la boucherie, des vaches laitières, des moutons, des mulets, de la laine, des cotonnades, es soieries, du fer, du cuivre, de l'étain, du plomb. Ils exportent de l'huile, des figues, des vêtements confectionnés, des cuirs, des ustensiles de ménage en bois, plats, cuillers, des poteries, du poivre rouge, de la bijouterie, des armes, de la toile de lin, des fruits, raisins, glands, caroubes, de la cire. » (Hanoteau et Letourneux 2003, p. 444).

\section{L'émigration temporaire de travail}

Les Kabyles sont réputés fort mobiles. Bien avant la colonisation française, ils sillonnaient une bonne partie de l'Afrique du Nord pour leur commerce mais aussi en quête de travail ; leur polyvalence les prédisposait à l'exercice de différents métiers. Ils louaient leurs bras comme soldats, maçons, constructeurs de moulins hydrauliques, faucheurs de blé, etc. de Kabylie occidentale) et M'ziti (originaires de Kabylie orientale) étaient nombreux à 
Alger et Constantine organisés en corporations, à l'instar d'autres groupes régionaux ou confessionnels algériens (Biskris, Mozabites, Juifs...) ; ils étaient employés comme terrassiers, jardiniers, gardes du Dey, etc. Rares étaient les Kabyles qui se fixaient définitivement loin de leurs villages; ce n'est qu'après la destruction des bases de l'économie kabyle traditionnelle lors des révoltes de 1857 et 1871 que l'émigration s'est transformée peu à peu en départs définitifs et lointains (Tunisie, Syrie, France...) (Cf. notice « Kabylie : l'émigration »).

\section{BIBLIOGRAPHIE}

BOURDIEU P., 1972, Esquisse d'une théorie de la pratique, précédé de trois études d'ethnologie kabyle, Genève, Droz.

CARETTE Cpt., 1848, Études sur la Kabylie proprement dite, Paris, Imprimerie Nationale.

DOUMANE S., 1993, Modernisation économique et pesanteur socio-culturelle en Algérie : l'exemple de la Kabylie, Thèse d'État, Université de Lille-I.

HANOTEAU A. et LeTOURneuX A., 2003 (réédition), La Kabylie et les coutumes kabyles, Tomes : I, II, III, Paris, Éditions Bouchène.

IBN KHALDOUN, 1999, Histoire des Berbères, Paris, Éditions Geuthner.

MAHÉ A., 2001, Histoire de la Grande Kabylie, XIX ${ }^{e}-X^{e}$ siècles. Anthropologie historique du lien social dans les communautés villageoises, Paris, Éditions Bouchène.

MAMMERI M., 1980, Poèmes kabyles anciens, p. 32, Paris, Maspéro.

MORIzOT J., 1985, Les Kabyles : propos d'un témoin, Paris, CHEAM.

\section{NOTES}

1. En réalité, à partir des années 1850 , la Kabylie était sinon occupée, du moins encerclée par l'armée française (1854 pour sa partie orientale et 1857 pour sa partie occidentale). Aussi, ses équilibres économiques étaient déjà sérieusement perturbés : installation des premiers villages de colonisation dans les vallées et piémonts (Tizi-Ouzou est fondée en 1858), premières mesures de séquestres fonciers lors des campagnes militaires de 1844-47, 1856-57 et de façon plus systématique en 1871, rupture du trafic caravanier avec les Hauts-Plateaux... Mais, jusqu'au début de la guerre d'indépendance (1954), on pouvait trouver encore quelques reliques de l'économie ancienne, notamment dans l'agriculture (arboriculture, jardinage...) et l'artisanat (poterie, vannerie...).

2. Selon J. Morizot (1985), citant des rapports de voyageurs du Moyen Âge jusqu'à l'avènement de la colonisation française (Marmol, Léon l'Africain, T. Shaw, des officiers de l'armée française...), les Kabyles ont réussi, bon an mal an, à atteindre des niveaux de production qui leur ont permis de faire face à leurs besoins essentiels et même de vendre des quantités substantielles de produits divers. 
3. Au début $\mathrm{du} \mathrm{xix}^{\mathrm{e}}$ siècle, alors que déclinaient les grandes cités où siégeaient les autorités ottomanes (la population d'Alger tomba à 30000 habitants, celle de Blida à 3600 et celle de Bougie à 2000 habitants...), beaucoup de villages de Kabylie comme ceux des Ait Yanni, voyaient leur population dépasser le millier d'habitants (4 à 6000 à Ait Lahcène selon J. Morizot).

4. Jusqu'à la fin du xix ${ }^{e}$ du siècle, excepté Bougie, il n'y avait point de villes en Kabylie; les centres urbains actuels étaient pour la plupart des villages agricoles crées pour les besoins de la colonisation sur le littoral et dans les vallées du Sébaou et de la Soummam : Tigzirt, Mirabeau, Maillot, Akbou...

5. L'exiguïté de la propriété foncière - moins de 10 hectares dans la plupart des cas-, ne nécessitait guère le recours au métayage et autres formes d'association dans la montagne kabyle alors que le khammessat* (association au cinquième de la récolte) était une pratique courante dans la plaine et les hauts plateaux algériens.

6. Il s'agit de petit élevage pour l'alimentation d'appoint (lait, viande...) car il n'y a guère de pâturages et de prairies en Kabylie montagnarde.

7. C'était le cas des Ait Abbas (Kabylie orientale) et de Djemâa n Saridj (Haut Sébaou) qui détenaient d'importantes forges, des Ait Yanni et des Ait Ouacif réputés pour leur orfèvrerie, des Iflissen n Lebher (Kabylie maritime) et des Ait Fraoucen (massif central) pour leur coutellerie et armurerie..., tandis que d'autres tribus comme les Ait Slimane du massif de Kendirou (Kabylie orientale) qui possédaient des mines de fer, les fournissaient en métal brut.

8. Chaque tribu avait son souk se tenant un jour par semaine et portant généralement le nom de la tribu précédé du nom du jour : Larbaâ $n$ At-Yiraten, Ssebt $n$ Amrawa, Letnayen At-Jennad...

9. J. Morizot rapporte que même après l'occupation de la Kabylie, l'administration française délivrait dans les années 1880, jusqu'à 10000 autorisations de circulation aux colporteurs de la seule confédération de tribus des Igawawen qui ne dépassait pas 80000 habitants.

\section{INDEX}

Mots-clés : Agriculture, Archéologie, Commerce, Economie, Emigration, Kabylie 